\title{
Microscopic Structure of the Wetting Film at the Surface of Liquid Ga-Bi Alloys
}

\author{
H. Tostmann, ${ }^{1}$ E. DiMasi, ${ }^{2}$ O. G. Shpyrko, ${ }^{1}$ P. S. Pershan, ${ }^{1}$ B. M. Ocko, ${ }^{2}$ and M. Deutsch ${ }^{3}$ \\ ${ }^{1}$ Division of Applied Sciences and Department of Physics, Harvard University, Cambridge, Massachusetts 02138 \\ ${ }^{2}$ Department of Physics, Brookhaven National Laboratory, Upton, New York 11973-5000 \\ ${ }^{3}$ Department of Physics, Bar-Ilan University, Ramat-Gan 52100, Israel
}

(Received 12 July 1999)

\begin{abstract}
$\mathrm{X}$-ray reflectivity measurements of the binary liquid Ga-Bi alloy reveal a dramatically different surface structure above and below the monotectic temperature $T_{\text {mono }}=222^{\circ} \mathrm{C}$. A Gibbs-adsorbed Bi monolayer resides at the surface in both regimes. However, a $30 \AA$ thick, Bi-rich wetting film intrudes between the

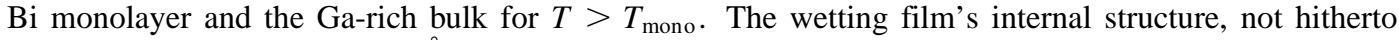
measured, is determined with $\AA$ resolution, showing a concentration gradient not predicted by theory and a highly diffuse interface with the bulk phase.
\end{abstract}

PACS numbers: 68.10.-m, 61.10.-i, 61.25.Mv, 68.45.Gd

A thick wetting film may be stable at the free surface of a binary immiscible liquid mixture. The temperature dependent formation of this surface film is strongly influenced by the bulk critical demixing of the underlying bulk phase [1]. In contrast to surface segregation, where a monolayer of the low surface tension component forms at the interface of the binary mixture, wetting is a genuine phase transition. It occurs at the interface resulting in the formation of a mesoscopically or a macroscopically thick film [2]. Until now, nearly all studies of wetting phenomena have been carried out with dielectric liquids dominated by long-range van der Waals interactions [3] and the experimental techniques used only achieve mesoscopic resolution. For binary alloys dominated by screened Coulomb interactions, evidence for a wetting transition has so far been obtained only for $\mathrm{Ga}-\mathrm{Bi}$ [4] and $\mathrm{Ga}-\mathrm{Pb}$ [5]. In the case of $\mathrm{Ga}-\mathrm{Bi}$, the formation of a Bi-rich wetting film has been detected by ellipsometry in the temperature range from 220 to $228^{\circ} \mathrm{C}$ [4]. However, since the film thickness is much smaller than the wavelength of visible light, ellipsometry cannot provide A-resolution structural information. By contrast, x-ray surface scattering techniques allow determination of the structure of the wetting film with atomic resolution and address issues such as the internal structure of the film and its evolution from molecular to mesoscopic thickness, none of which were hitherto addressed by any of the previous measurements.

The wetting phase transition is depicted schematically in Fig. 1. Between the monotectic temperature $T_{\text {mono }}$ and the bulk critical temperature of demixing $T_{\text {crit }}$, the bulk phase separates into two immiscible phases, the high density Bi-rich phase and the low density Ga-rich phase. Below the characteristic wetting temperature $T_{w}<T_{\text {crit }}$, the high density phase is confined to the bottom of the container [see Fig. 1(a)]. For Ga-Bi, $T_{w}$ coincides with $T_{\text {mono }}$ [4]. Above $T_{w} \approx T_{\text {mono }}$ the high density phase completely wets the free surface by intruding between the low density phase and the gas phase in defiance of gravity [see Fig. 1(b)] [2,7].
The Ga-Bi alloy was prepared in an inert-gas box using $>99.9999 \%$ pure metals. A solid Bi ingot was placed in a Mo pan and liquid $\mathrm{Ga}$ was added to cover the $\mathrm{Bi}$ ingot. At room temperature, the solubility of $\mathrm{Bi}$ in $\mathrm{Ga}$ is less than 0.2 at. $\%$. Increasing the temperature results in continuously dissolving more $\mathrm{Bi}$ in the Ga-rich phase with solid $\mathrm{Bi}$ remaining at the bottom of the pan up to the monotectic temperature, $T_{\text {mono }}=222{ }^{\circ} \mathrm{C}$. The initial Bi content was chosen to be high enough that the two-phase equilibrium along the miscibility gap could be followed up to a few degrees below $T_{\text {crit }}$ without crossing into the homogeneous phase region (see Fig. 1). The temperature on the sample surface was measured with a Mo coated thermocouple. The alloy was contained in an ultrahigh vacuum chamber and the residual oxide on the sample was removed by sputtering with $\mathrm{Ar}^{+}$ions [8]. Surface sensitive X-ray

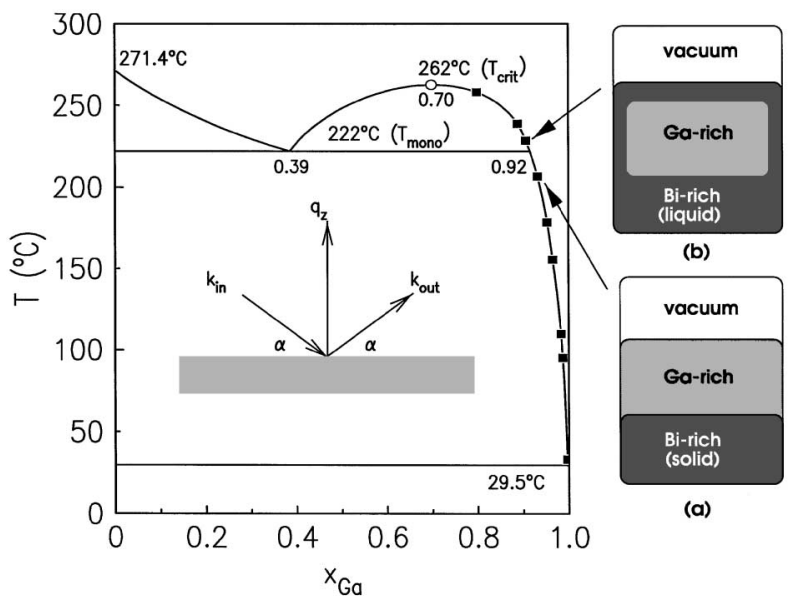

FIG. 1. Phase diagram of Ga-Bi (Ref. [6]). Between 29.5 and $222^{\circ} \mathrm{C}$, solid $\mathrm{Bi}$ is in equilibrium with a Ga-rich liquid phase. Between 222 and $262^{\circ} \mathrm{C}$, a liquid Bi-rich phase is in coexistence with a liquid Ga-rich phase. The surface structure of this alloy has been measured between 35 and $258^{\circ} \mathrm{C}$ at selected points along the coexistence line ( $\boldsymbol{\square})$. For $T<222^{\circ} \mathrm{C}$ partial wetting occurs (a), whereas complete wetting is found for $T>$ $222^{\circ} \mathrm{C}(\mathrm{b})$. The inset depicts the geometry for specular $\mathrm{x}$-ray reflectivity. 
reflectivity (XR) experiments were carried out using the liquid surface spectrometer at beam-line X22B at the National Synchrotron Light Source with an $\mathrm{X}$-ray wavelength $\lambda=1.24 \AA$ and a detector resolution of $\Delta q_{z}=0.03 \AA^{-1}$. The background intensity, due mainly to scattering from the bulk liquid, was subtracted from the specular signal by displacing the detector out of the reflection plane [8].

The intensity reflected from the surface, $R\left(q_{z}\right)$, is measured as a function of the normal component $q_{z}=$ $(4 \pi / \lambda) \sin \alpha$ of the momentum transfer. The XR geometry is depicted schematically in the inset of Fig. 1. $R\left(q_{z}\right)$ yields information about the surface-normal structure as given by

$$
R\left(q_{z}\right)=R_{F}\left(q_{z}\right)\left|\Phi\left(q_{z}\right)\right|^{2} \exp \left[-\sigma_{\mathrm{cw}}^{2} q_{z}^{2}\right],
$$

where $R_{F}\left(q_{z}\right)$ is the Fresnel reflectivity of a flat, infinitely sharp surface, and $\Phi\left(q_{z}\right)$ is the Fourier transform of the local surface-normal density profile $\tilde{\rho}(z)[8,9]$ calculated using the distorted wave Born approximation (DWBA) [10]:

$$
\Phi\left(q_{z}\right)=\frac{1}{\rho_{\infty}} \int d z \frac{d \tilde{\rho}(z)}{d z} \exp \left(l \sqrt{q_{z}^{2}-q_{\text {crit }}^{2}} z\right),
$$

with the bulk electron density $\rho_{\infty}$ and the critical wave vector $q_{\text {crit }}$. The exponential factor in Eq. (1) accounts for roughening of the intrinsic density profile $\tilde{\rho}(z)$ by capillary waves $(\mathrm{CW})$ :

$$
\sigma_{\mathrm{cw}}^{2}=\frac{k_{B} T}{2 \pi \gamma} \ln \left(\frac{q_{\mathrm{max}}}{q_{\mathrm{res}}}\right),
$$

where $\gamma$ is the macroscopic surface tension of the interface between the vacuum and the liquid alloy. The CW spectrum is cut off at small $q_{z}$ by the detector resolution $q_{\text {res }}$ and at large $q_{z}$ by the atomic size $a$ with $q_{\max } \approx \pi / a$ [8]. However, $\tilde{\rho}(z)$ cannot be obtained directly from the measured reflectivity as given by Eq. (1) due to the loss of phase information. We resort therefore to the widely accepted procedure of adopting a physically motivated model for $\tilde{\rho}(z)$ and fitting its Fourier transform to the experimentally determined $R\left(q_{z}\right)$, as will be shown below [8].

The reflectivity of pure liquid Ga exhibits a pronounced interference peak indicating surface-induced layering of ions near the surface [see Fig. 2(a)] [11]. Previous experiments on $\mathrm{Ga}-\mathrm{Bi}$ at low temperatures $[12,13]$ show that the layering peak is suppressed in the liquid alloy and $R\left(q_{z}\right)$ is dominated by a broad maximum consistent with a single $\mathrm{Bi}$ monolayer segregated at the surface as expected since $\gamma$ is considerably lower for $\mathrm{Bi}$ than for $\mathrm{Ga}$ [13].

Here, we report x-ray measurements from liquid Ga-Bi for $T$ up to $258{ }^{\circ} \mathrm{C}$ encompassing the formation of the wetting film at $T>T_{\text {mono }}$, the lowest temperature at which the liquid Bi-rich phase is stable. The discussion of the data is divided into two parts: (i) $T<T_{w} \simeq T_{\text {mono }}$ (110-207 ${ }^{\circ} \mathrm{C}$ ), and (ii) $T>T_{w}\left(228-258^{\circ} \mathrm{C}\right)$. We present the results for $T<T_{\text {mono }}$ [scenario (a) in Fig. 1] first since this corresponds to the simpler surface structure with monolayer segregation but no wetting film.
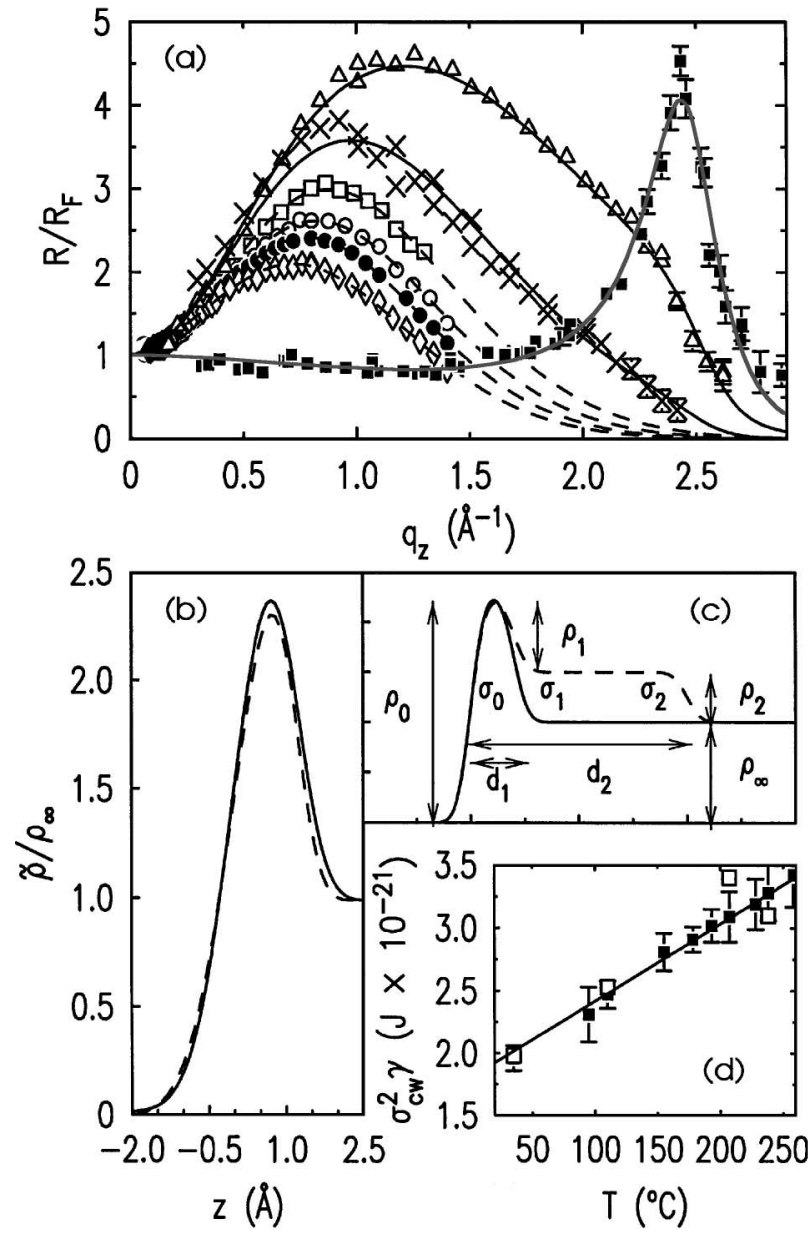

FIG. 2. (a) Fresnel normalized reflectivity $R / R_{F}$ from Ga-Bi alloys for $T<T_{\text {mono: }}(\boldsymbol{\square}): 100$ at. $\% \mathrm{Ga}$; $(\triangle): 99.7 \% \mathrm{Ga}$ $\left(35^{\circ} \mathrm{C}\right) ;(\times): 98.7 \%$ Ga $\left(95^{\circ} \mathrm{C}\right) ;(\square): 98.4 \%$ Ga $\left(110{ }^{\circ} \mathrm{C}\right)$; (○): $96.5 \%$ Ga $\left(155^{\circ} \mathrm{C}\right)$; $(\bullet): 95.3 \% \mathrm{Ga}\left(178^{\circ} \mathrm{C}\right)$ and $(\diamond)$ : $93.2 \% 1 \mathrm{Ga}\left(207^{\circ} \mathrm{C}\right)$. Shaded line: fit to layered density profile of pure Ga (see Ref. [11]); solid lines: fit to layered density profile of Ga plus Bi monolayer (see Ref. [12]); broken lines: fit to $\mathrm{Bi}$ monolayer (this work). (b) Intrinsic density profiles normalized to $\rho_{\infty}$ for $\mathrm{Ga}-\mathrm{Bi}$ for $110^{\circ} \mathrm{C} \leq T \leq 207^{\circ} \mathrm{C}$ using the one-box model described in the text. The profiles essentially fall on top of each other and two representative fits are shown: $110{ }^{\circ} \mathrm{C}$ (solid line) and $207^{\circ} \mathrm{C}$ (broken line). (c) Schematic representation of the (two-)box model. (d) $\mathrm{CW}$ roughness $\sigma_{\mathrm{cw}}$ for $\mathrm{Ga}-\mathrm{Bi}$ alloys as a function of temperature using $\gamma$ from our fit (ם) and from macroscopic surface tension measurements ( $\square$, see Ref. [14]).

Below $T_{\text {mono. }}$-Figure 2(a) shows $R\left(q_{z}\right)$ from liquid $\mathrm{Ga}-\mathrm{Bi}$ at selected points along the coexistence line between 110 and $207^{\circ} \mathrm{C}$. Subsurface layering is impossible to resolve at these temperatures, and we compare the data to the reflectivity calculated in the framework of the DWBA using a simple model to describe the surface structure: the near-surface density, which is dominated by the Bi monolayer segregation, is represented by one box of density $\rho_{1}$ and width $d_{1}$. The density profiles are shown in Fig. 2(b) and the box model is shown schematically in Fig. 2(c) (solid line). The best fit to $R\left(q_{z}\right)$ that corresponds 
to this density profile is represented by the broken lines in Fig. 2(a). The mathematical description of the general box model with a maximum number of two boxes is

$$
\begin{aligned}
\tilde{\rho}(z)= & \frac{\rho_{0}}{2}\left\{1+\operatorname{erf}\left(\frac{z}{\sigma_{0}}\right)\right\} \\
& -\sum_{i=1}^{2} \frac{\rho_{i}}{2}\left\{1+\operatorname{erf}\left(\frac{z-d_{i}}{\sigma_{i}}\right)\right\},
\end{aligned}
$$

where $\sigma_{0}$ and $\sigma_{i}$ are the intrinsic roughnesses of each of the three interfaces. The total roughness, $\sigma$, of the interface between the vapor and the outermost surface layer is given by $\sigma^{2}=\sigma_{0}^{2}+\sigma_{\mathrm{cw}}^{2}$ with $\sigma_{0}=0.78 \pm 0.15 \AA$ for $T<T_{\text {mono }}$. As predicted by capillary wave theory, the product $\sigma_{\mathrm{cw}}^{2} \gamma$ (with $\gamma$ from our fits) depends linearly on $T$ [see Eq. (3) and (ם) in Fig. 2(d)] over the entire temperature range. This variation is essentially the same if $\gamma$ is not taken from our fits but from macroscopic surface tension measurements [ $\square$ in Fig. 2(d)] [14]. Apart from the increasing surface roughness, the structure of the surface does not change over the entire temperature range from 110 to $207^{\circ} \mathrm{C}$, as witnessed by the intrinsic density profiles that fall right on top of each other once the theoretically predicted temperature dependence of the $\mathrm{CW}$ roughness has been corrected for [Fig. 2(b)]. Between 212 and $224^{\circ} \mathrm{C}$, we observed rapid and random changes in the scattered intensity over the entire $q_{z}$ range. This unstable behavior of the surface is most likely due to the coexistence of patches of different film thickness induced by the $\sim 6 \mathrm{~K}$ surface-normal temperature gradient.

Above $T_{\text {mono. }}$ - The reflectivity stabilized above $T_{\text {mono }}$ at about $228{ }^{\circ} \mathrm{C}$ and a sharper peak in $R\left(q_{z}\right)$ appeared centered around $0.13 \AA^{-1}$ (see Fig. 3). This prominent structural feature is a clear indication that a high density thick film has formed at the surface. Similarly, the persistence of the broad maximum centered around $q_{z} \approx 0.75 \AA^{-1}$ indicates that $\mathrm{Bi}$ monolayer segregation is still present along with the newly formed thick wetting film. Several models were used to fit $R\left(q_{z}\right)$ but they all result in essentially the same density profiles. Here, we use the simple two-box model [see broken line in Fig. 2(c) and Eq. (4)]. As can be seen in Fig. 3, this simple model gives an excellent description of the experimentally obtained reflectivity. The pertinent density profiles describing the surface-normal structure of Ga-Bi alloys for $T>T_{\text {mono }}$ are shown in the inset of Fig. 3 and have the following features that can be compared to present predictions of wetting theory: (i) density, (ii) thickness, and (iii) roughness of the wetting film.

(i) The concentration profile is highly inhomogeneous with a high density region at the outermost surface layer [15]. The integrated density of this adlayer is consistent with a monolayer of the same density as the monolayer found for $T<T_{\text {mono. However, the Bi monolayer segre- }}$ gated on top of the wetting film is rougher and broader than the Bi monolayer segregated on top of the Ga-rich bulk

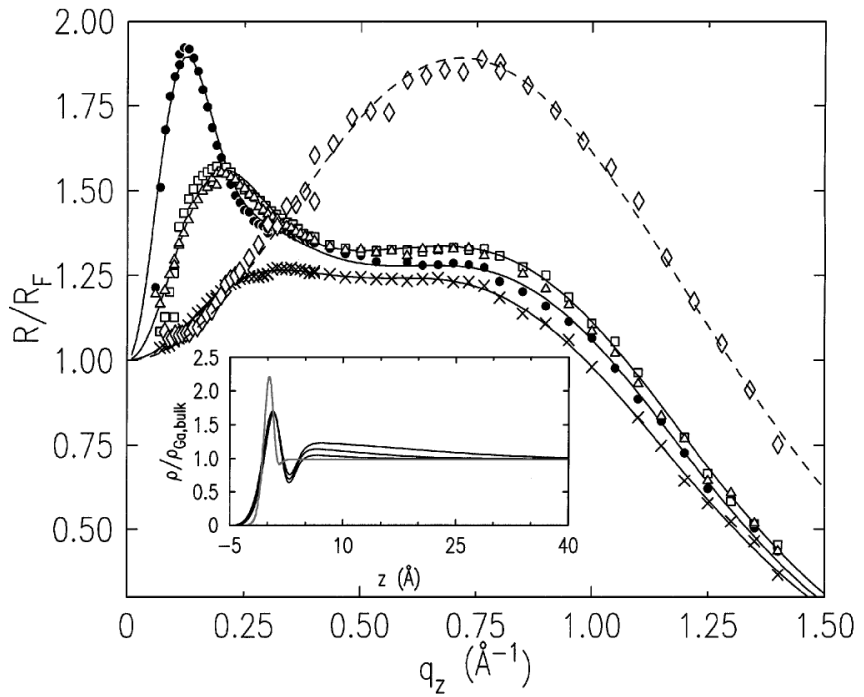

FIG. 3. $R / R_{F}$ from Ga-Bi alloys for $T>T_{w}:(\bullet): 90.6$ at. $\%$ $\mathrm{Ga}\left(228^{\circ} \mathrm{C}\right) ;(\square): 88.8 \%$ Ga $\left(238^{\circ} \mathrm{C}\right) ;(\triangle)$ : ditto, but after heating to $258{ }^{\circ} \mathrm{C}$ and cooling down; $(\times): 79.1 \%$ Ga $\left(258^{\circ} \mathrm{C}\right)$; [ for comparison, see $(\diamond), 207^{\circ} \mathrm{C}$ ]. Solid lines fit to two-box model; broken line: fit to one-box model. Inset: intrinsic density profiles, $\tilde{\rho}(z)$, for $\mathrm{Ga}-\mathrm{Bi}$ alloys normalized to the bulk density, $\rho_{\mathrm{Ga}, \text { bulk }}=\rho_{\infty}$, at $T>T_{w}$ : solid lines ordered in decreasing wetting film density: $228^{\circ} \mathrm{C}, 238^{\circ} \mathrm{C}$, and $258^{\circ} \mathrm{C}$. Compare to the one-box density profile of $\mathrm{Ga}-\mathrm{Bi}$ at $207^{\circ} \mathrm{C}$ (shaded line).

phase for $T<T_{\text {mono }}$. This change in surface structure corresponds to a change in the intrinsic roughness of the interface liquid/vacuum, $\sigma_{0}$, from $0.78 \pm 0.15 \AA$ below $T_{\text {mono }}$ to $1.7 \pm 0.3 \AA$ above $T_{\text {mono }}$. However, the $\mathrm{CW}$ roughness still follows the predicted $T$-dependent slope predicted by Eq. (3) [see Fig. 2(d)]. This change of $\sigma_{0}$ upon crossing $T_{\text {mono }}$ is possibly related to the fact that, for $T>T_{\text {mono }}$, the pure Bi monolayer segregates against a Bi-rich wetting film made out of atoms of different size and with repulsive heteratomic interactions. By contrast, for $T<T_{\text {mono }}$, this segregation takes place against almost pure Ga. The fact that the Bi monolayer segregation persists from partial wetting to complete wetting means that the wetting film does not intrude between the Ga-rich bulk phase and vacuum, as assumed by wetting theory, but between the Bi monolayer already segregated at the surface and the Ga-rich bulk phase. This should have a pronounced influence on the energy balance at the surface which ultimately governs the wetting phase transition. Even though the possibility of an unspecified concentration gradient is included in Cahn's general theory [1], to our knowledge an inhomogeneous density profile is neither treated explicitly in theoretical calculations (see the homogeneous profiles displayed in $[16,17])$ nor found experimentally. At $228^{\circ} \mathrm{C}$, the density of the thick wetting film is $\rho_{2}=(1.25 \pm 0.03) \rho_{\infty}$, where $\rho_{\infty}$ is the density of the Ga-rich bulk phase. This agrees very well with the ratio of the densities of the coexisting Bi-rich and Ga-rich phases in the bulk, calculated from the phase diagram to be 1.23 . The density of the wetting film reaches $\rho_{\infty}$ with increasing $T\left(\rho_{2} / \rho_{\infty}=1.18 \pm 0.03\right.$ at 
$238^{\circ} \mathrm{C}$ and $1.08 \pm 0.02$ at $258^{\circ} \mathrm{C}$ ) as the densities of the two bulk phases converge upon approaching $T_{\text {crit }}$ (their density ratio is 1.20 at $238{ }^{\circ} \mathrm{C}$ and 1.08 at $258^{\circ} \mathrm{C}$ ). This strongly supports the conclusion that the thick wetting film is the Bi-rich bulk phase as predicted by wetting theory.

(ii) Without fitting, the thickness $d_{2} \approx 2 \pi / q_{\text {peak }}$ of the wetting film is estimated from the maximum position of the peak, $q_{\text {peak }}$, to be about $25 \AA$ at $228^{\circ} \mathrm{C}$. From fitting, the thickness that best represents the data at $228^{\circ} \mathrm{C}$ is $30 \AA+20 /-10 \AA$. The confidence limits arise primarily from systematic errors associated with temperature inhomogeneities and sample curvature. The latter limits the ability to gather data at the smallest angles. The thickness of the wetting film is also corroborated by independent grazing incidence diffraction experiments resolving the in-plane structure which will be reported elsewhere [18]. The wetting film in this Coulomb liquid with short-range interactions is considerably thinner than wetting films that have been observed in dielectric liquids with long-range interactions [3,19]. An important question concerning the thickness of a wetting film is whether the wetting film has been investigated in equilibrium. The fact that identical reflectivities were measured at $238^{\circ} \mathrm{C}$ taken $24 \mathrm{~h}$ apart (Fig. 3) is strong evidence that the film thickness is in equilibrium in our study [19].

(iii) The roughness between the Bi-rich wetting film and the Ga-rich bulk is much higher than the roughness of the free interface liquid metal/vacuum. This is be expected since the interfacial tension between two similar liquids is generally much lower than the liquid/gas surface tension [20]. On the other hand, it is not clear how sharp a concentration gradient should be expected between the Ga-rich and the Bi-rich phase and the interface may be essentially diffuse, over a length scale much larger than the $\mathrm{CW}$ fluctuations. With increasing temperature, the wetting film becomes less well defined and it is not obvious whether this is due to the fact that the film is becoming slightly thinner or if only the interface between two converging phases gets more diffuse. Up to about $258^{\circ} \mathrm{C}$, we have not detected any thickening of the wetting film due to the increasing correlation length of concentration fluctuations indicating that we are still far away from the temperature where critical fluctuations directly affect the wetting film.

In summary, we investigated the structural changes occurring on atomic length scales at the surface of liquid $\mathrm{Ga}-\mathrm{Bi}$ during the wetting transition. In the case of partial wetting $\left(T<T_{\text {mono }}\right)$, a Bi monolayer segregates at the surface to lower the surface energy. Above the monotectic temperature, complete wetting is found and a wetting film that is at least $30 \AA$ thick intrudes between this monolayer and the Ga-rich bulk phase. This is the first time that the microscopic structure of a wetting film has been studied and that the concentration profile of the wetting film has been shown to vary on a level of several atomic diameters.

This work is supported by the U.S. DOE Grant No. DEFG02-88-ER45379, and the U.S.-Israel Binational Science Foundation, Jerusalem. Brookhaven National Laboratory is supported by U.S. DOE Contract No. DE-AC0298CH10886.

[1] J. W. Cahn, J. Chem. Phys. 66, 3667 (1977).

[2] S. Dietrich, Physica (Amsterdam) 168A, 160 (1990).

[3] S. Dietrich, in C. Domb and J. L. Lebowitz, Phase Transitions and Critical Phenomena (Academic Press, New York, 1988), Vol. 12.

[4] D. Nattland, P. D. Poh, S. C. Müller, and W. Freyland, J. Non-Cryst. Solids 205-207, 772 (1996).

[5] D. Chatain and P. Wynblatt, Surf. Sci. 345, 85 (1996).

[6] T. B. Massalski et al., Binary Alloy Phase Diagrams (ASM International, Materials Park, OH, 1990).

[7] J. S. Rowlinson and B. Widom, Molecular Theory of Capillarity (Clarendon Press, Oxford, 1982).

[8] H. Tostmann, E. DiMasi, P. S. Pershan, B. M. Ocko, O. G. Shpyrko, and M. Deutsch, Phys. Rev. B 59, 783 (1999).

[9] P. S. Pershan and J. Als-Nielsen, Phys. Rev. Lett. 52, 759 (1984).

[10] X. L. Zhou, Phys. Rev. E 52, 1938 (1995).

[11] M. J. Regan et al., Phys. Rev. Lett. 75, 2498 (1995).

[12] H. Tostmann et al., Ber. Bunsen-Ges. Phys. Chem. 102, 1136 (1998).

[13] N. Lei, Z. Huang, and S. A. Rice, J. Chem. Phys. 104, 4802 (1996).

[14] K. B. Khokonov et al., Elektrokhimiya 10, 911 (1974); H. U. Tschirner et al., Wiss. Z. Tech. Hochsch. Karl-MarxStadt 26, 640 (1984).

[15] The dip in density between the outermost Bi adlayer and the thick wetting layer can be modeled with an atomic model similar to the one used for $\mathrm{Bi}$ segregated on top of Ga with subsurface layering (see Ref. [12]). Including subsurface layering provides a slightly improved fit but does not substantially alter the parameters of the wetting layer.

[16] For example, see p. 75 in Ref. [3] or p. 218 in Ref. [6].

[17] D. Beysens and M. Robert, J. Chem. Phys. 87, 3056 (1987).

[18] E. DiMasi et al., J. Phys. Condens. Matter (to be published).

[19] R. F. Kayser, M. R. Moldover, and J. W. Schmidt, J. Chem. Soc. Faraday Trans. 2 82, 1701 (1986).

[20] J. Israelachvili, Intermolecular \& Surface Forces (Academic Press, New York, 1992). 